\title{
Telomeres, cardiovascular aging, and potential intervention for cellular senescence
}

\author{
ZHANG WeiLi ${ }^{*}$, HUI RuTai \& YANG ShuJun \\ State Key Laboratory of Cardiovascular Diseases, Fuwai Hospital, National Center for Cardiovascular Diseases, Chinese Academy of \\ Medical Sciences and Peking Union Medical College, Beijing 100037, China
}

Received May 25, 2014; accepted June 14, 2014

\begin{abstract}
A consistent association has been observed between leukocyte telomere length (LTL) and atherosclerosis, but the mechanisms underlying these associations are still not well understood. Premature biology aging was evident in atherosclerotic plaques, characterized by reduced cell proliferation, irreversible growth arrest and apoptosis, and telomere attrition. As atherosclerosis is a state of chronic low-grade inflammation and increased oxidative stress, shortened LTL in patients with atherosclerosis might stem from the two sources, one is an accelerated rate in hematopoietic stem cells (HSCs) replication to replace leukocytes consumed in the inflammatory process, and another is the increase in the loss of telomere repeats per replication. Thus, diminished HSC reserves at birth and age-dependent telomere attrition afterward are mirrored in shortened LTL during the adulthood. In addition, the inter-individual variation of LTL in the general population can be partly explained by genetic factors regulating telomere maintenance and the rate of HSCs replication. Atherosclerosis is an aging-related disease, and practically all humans develop atherosclerosis if they live long enough. Here we overview the potential roles of LTL dynamics in the imbalance between injurious oxidative stress/inflammation and endothelial repair during the pathogenesis of age-related atherosclerosis, and discuss the possibility that preventing accelerated cellular senescence is a potential target in prevention of atherosclerosis.
\end{abstract}

telomere, aging, atherosclerosis, vascular cell senescence

Citation: Zhang WL, Hui RT, Yang SJ. Telomeres, cardiovascular aging, and potential intervention for cellular senescence. Sci China Life Sci, 2014, 57: 858-862, doi: $10.1007 / \mathrm{s} 11427-014-4700-8$

Aging, which is broadly defined as the time-dependent functional decline, has attracted curiosity and imagination throughout the history of humankind. Atherosclerosis and cardiovascular diseases are associated with advanced age, and premature vascular aging can be accelerated by cardiovascular risk factors, including smoking, alcohol intake, diabetes, and dyslipidemia. However, at an individual level there is wide variation in the development of atherosclerosis, even in individuals with the similar risk factor profiles. The reasons why there are inter-individual heterogeneities are still incompletely understood, but telomere attrition is rec-

*Corresponding author (email: zhangweili1747@yahoo.com) ognized to represent one molecular mechanism underlying biological aging and cellular senescence [1].

Telomeres are the TTAGGG nucleotide repeats at the ends of mammalian chromosomes, which preserve chromosome stability and integrity [2]. Telomeres are progressively shortened with each replication of somatic cells. When telomere lengths are shortened to a critical value, they lose capping function at the chromosomal ends, resulting in activation of DNA damage checkpoints and cell senescence. Emerging epidemiological and experimental evidence has linked leukocyte telomere length (LTL) with vascular cell senescence and risk of atherosclerosis and cardiovascular diseases [3]. However, the question remains 
unanswered regarding whether telomere length is merely a biomarker of atherosclerosis, or an active player in its pathogenesis, or both. Here, this review summarizes the mechanisms underlying telomere mediated hematopoietic senescence and vascular aging in atherosclerosis, and the potential therapeutics for cellular senescence.

\section{Normal and premature vascular aging}

Vessel aging, even in the absence of atherosclerosis, can lead to vascular remodeling characterized by intimal and medial thickening, endothelia cell dysfunction, increased collagen deposition, decreased elastin content, and impaired vessel elasticity, which hence results in arterial stiffness and related vascular diseases. In addition, aged vessels also show increased secretion of proinflammatory cytokines and uptake of plasma lipoproteins, resulting in persistent vascular inflammation [4]. Thus, the effects of atherosclerosis are superimposed on normal aging of vessels.

Cell senescence is defined as the irreversible loss of the ability of cells to divide, including replicative senescence and stress-induced premature senescence (SIPS). Replicative senescence occurs with exhaustion of proliferative lifespan over time, and is associated with critically shortened telomeres at chromosome ends. In contrast, SIPS is not usually characterized by telomere shortening and triggered by external stimuli, such as oxidizing agents and radiation, which activate the intracellular senescence cascade prematurely. Senescent cells are characterized by "specific" markers, senescence-associated $\beta$ galactosidase (SA $\beta \mathrm{G})$, and altered expression of cell cycle regulators. Increased numbers of SA $\beta$ G-positive vascular smooth cells (VSMCs), endothelial cells (ECs), and monocyte/macro- phages are observed in aged vessels and atherosclerotic lesions [5,6], reinforcing the idea that atherosclerosis is associated with premature cellular senescence.

Does biological aging promote atherosclerosis, or does atherosclerosis promote vessel aging and cellular senescence? Obviously, they are not mutually exclusive and both occur simultaneously. Recent evidence indicates that peripheral leukocyte telomere length can be used as a systemic marker for cellular senescence and aging-related diseases, which might yield new insights into the underlying molecular mechanisms of vascular aging and improve cardiovascular risk stratification.

\section{Telomere shortening}

Telomere shortening is observed during normal aging and premature aging. Telomere length and integrity is mainly regulated by telomerase, an enzyme composed of a telomerase RNA component (TERC) and telomerase reverse transcriptase (TERT). Generally, telomerase is active only in germ lines, during embryogenesis, in adult stem cells and in activated immune cells, while most somatic cells repress TERT expression at the transcriptional level, suggesting that its activity is tightly regulated during development and differentiation. Although telomere length results from a dynamic balance between elongation and shortening of the chromosome ends, mean telomere length within cells is a direct indication of the level of telomerase activity. And at birth, telomere lengths in most tissues from the same subject are similar [7].

Telomeres are bound by a complex of proteins known as Shelterin, including TRF1, TRF2, Rap1, TIN2, PTOP, and POT1, which functions to prevent the access of DNA repair proteins to the telomeres. Otherwise, telomeres would be "repaired" as DNA breaks leading to chromosome fusions. Due to their restricted DNA repair, DNA damage at telomeres is notably persistent and thus more likely to induce cell senescence and/or apoptosis [8].

Shortened telomeres are evident in atherosclerosis, observed in plaque VSMCs and ECs relative to the normal vessel wall, and in circulating EPCs [9]. Shorter telomeres and low levels of telomerase expression and activity are functionally important in VSMC senescence, because ectopic telomerase expression can increase lifespan of both plaque and normal VSMCs. In addition, arterial segments resistant to atherosclerosis, such as internal mammary artery or ascending aorta, have longer telomeres than the aortic regions prone to the disease, and the loss of telomeric DNA in the aortic regions was found to become more dramatic with increasing donor age [10]. This difference can be partly explained by repeated hemodynamic stress to endothelium in the aortic vessels, which is the initiating event to vascular dysfunction. The internal thoracic artery is known to be subjected to less hemodynamic stress and thus free of atherosclerotic plaques even among the elderly. Besides hemodynamic injury, it will be of interest to investigate whether the existence of intrinsic genetic or developmental variations in telomere regulation also underlies location-specific predisposition in atherogenesis.

\section{Leukocyte telomere length (LTL) and athe- rosclerosis}

Telomere length displays a large inter-individual variation at birth and throughout the human lifespan, which is influenced by heredity, gender, race, and environmental exposures. Recent studies have shown that birth LTL is a major determinant of LTL at any age, as persons who are born with short LTL are likely to display short LTL later in life. Thus, individuals who inherited shorter telomeres may display shorter systemic and vascular telomeres, and are at an increased risk for early senescence of vascular tissue, causing atherosclerosis. This implies that those subjects with high-risk predisposition to atherosclerosis and cardiovascu- 
lar diseases could be identified in their early life.

Emerging epidemiological studies have shown a consistent association between leukocyte telomeres and atherosclerosis. Telomere length in leukocytes is shorter in patients with clinical and subclinical features of atherosclerosis compared with control subjects, after adjustment for age, sex, and race [11-13]. The study by Zhang et al. [14] provided novel evidence that telomere attrition contributes to the presence of both atherothrombotic and hemorrhagic stroke in Chinese patients, independent of traditional vascular risk factors. Moreover, their study showed that atherothrombotic stroke patients with shorter telomeres had worse prognosis of post-stroke death during the long-term follow-up. Studies examining the LTL-mortality relation in same-sex elderly twins have found that the co-twins with the shorter LTL were more likely to die first during the follow-up period [15]. In addition, persons with multiple vascular risk factors such as high BMI, sedentary lifestyle, insulin resistance or cigarette smoking, also display relatively shorter LTL, suggesting that in part the rate of LTL shortening is modified by environmental factors.

Atherosclerosis is an aging-related disease which depends on the balance between the injurious and repair elements. The injurious element is partially due to the accumulated burden of oxidative stress and chronic low-grade inflammation on the vascular endothelium, which can accelerate the pace of age-dependent LTL shortening [16]. Another potential mechanism that explains the LTLerosclerosis relation focuses on vascular repair, which is largely implemented by endothelial progenitor cells (EPCs) originating from the hematopoietic stem cells (HSCs) pool. HSCs have insufficient telomerase activity to add telomere repeats onto the ends of chromosomes and prevent telomere shortening in leukocytes. Thus, diminished HSC reserves at birth, their accelerated attrition rate afterward, or both, are expressed in the form of shortened LTL during adulthood, which confers increased risk for atherosclerosis. This risk is largely attributed to insufficient circulating numbers and reduced replicative function of EPCs. Consistently, the number and proliferative potential of circulating EPCs are lower in atherosclerosis and in older person [17]. In this way, telomere length in HSCs is both a biomarker of atherosclerosis and a determinant of its development.

\section{Genetics of leukocytes telomere length}

Studies in twins, siblings and families have found that LTL is heritable with estimated heritability of $0.36-0.84$ [18]. Thus, genes that are directly involved in telomere maintenance and those whose function impacts HSC replication kinetics may be associated with telomere-related diseases. For example, major mutations in TERT and TERC genes cause telomerase deficiency and lead to premature development of rare monogenic diseases, such as pulmonary fi- brosis, liver cirrhosis, aplastic anemia, and tumors [19]. However, the specific and rare mutations that cause these diseases do not explain the wide inter-individual variation of LTL in the general population. Only recent genome-wide association studies (GWAS) have identified that single-nucleotide polymorphisms (SNPs) in loci that harbor oligonucleotide/oligosaccharide-binding fold containing 1 gene $(O B F C 1), T E R C$ and the chemokine (C-X-C motif) receptor 4 gene $(C X C R 4)$ are associated with LTL [20,21].

TERC plays a central role in telomere biology. $O B F C 1$ is a newly discovered telomere maintenance gene in humans, which functions in the negative regulation of telomerase [22]. CXCR4 regulates neutrophil release from the bone marrow and plays a key role in the damage-repair feedback loop between HSCs and the endothelium, which regulates the signals that guide the recruiting of EPCs to the area of vascular injury [23]. CXCR4 variants might attenuate the rate of HSC replication by slowing the mobilization of neutrophils from the bone marrow and thus resulting in a longer LTL. Importantly, CXCR4 modulates inflammatory response in atherosclerosis [24], and its cognate ligand CXCL12 locus was found by GWAS to be associated with myocardial infarction [25]. However, it should be noted that LTL-regulated genes that have been discovered through GWAS explain only a small portion of the inter-individual variation in LTL [26].

\section{Therapy targeting aging in atherosclerosis}

The increasing evidence that accelerated premature cell senescence occurs in vascular disease has given an impetus to search for treatments that can promote longevity and delay senescence $[27,28]$. Currently, it is debatable whether telomere length and telomerase are targets in atherosclerosis, although agents that promote telomere stability in vessel cells may be beneficial. First, although atherosclerosis is associated with telomere shortening in vascular cells, the evidence is lacking to demonstrate that shortening occurs to a critical length that impair function, as the rate of LTL shortening is rapid during the early life and remains relatively stable during the adulthood (between 20 and 70 years of age) when vascular aging is apparent [29]. Second, further evidence is needed to reveal whether telomere shortening initiates atherosclerosis rather than being a feature of advanced plaques. Although the lifespan of VSMCs and ECs can be extended by ectopic expression of telomerase, it is not clear if this is due to effects only on telomere length or other causes for their senescence [30]. In addition, the animal model studies of telomerase manipulation in atherosclerosis are contradictory. Finally and most importantly, increasing telomerase expression systemically has the potential risk to be carcinogenic.

As the rate of age-dependent telomere shortening is accelerated by accumulated burden of oxidative stress and 
inflammation, a number of currently available drugs and compounds are likely to indirectly delay premature aging by attenuating ROS and oxidative DNA damage. Such examples include antioxidants, statins, and angiotensin-converting enzyme (ACE) inhibitors and angiotensin receptor blockers (ARBs). Dietary status also has potential to reduce vascular aging, cellular senescence, and atherosclerosis.

\section{Conclusion}

In summary, atherosclerosis is a state of chronic imbalance in which the injurious effect of oxidative stress/inflammation exceeds endothelial repair capacity of HSCs and EPCs, which largely depend on telomere length, as expressed in LTL. Both normal vascular aging and atherosclerosis are associated with cellular senescence. Thus, shortened LTL can predict increased atherosclerotic risk as a biomarker of atherosclerosis and a determinant of its development. How important would it be to record the individual's LTL dynamics from birth to adulthood to old age? It depends on whether optimal methods of LTL measurement will be developed for clinical use, and whether preventive and/or therapeutic steps can be guided by LTL results, given that advanced atherosclerosis is likely to manifest irreversible changes.

1 Calado RT, Young NS. Telomere diseases. N Engl J Med, 2009, 361: 2353-2365

2 Blackburn EH. Structure and function of telomeres. Nature, 1991, 350: $569-573$

3 Nilsson PM, Tufvesson H, Leosdottir M, Melander O. Telomeres and cardiovascular disease risk: an update 2013. Transl Res, 2013, 162: 371-380

4 O'Rourke MF, Hashimoto J. Mechanical factors in arterial aging: a clinical perspective. J Am Coll Cardiol, 2007, 50: 1-13

5 Matthews C, Gorenne I, Scott S, Figg N, Kirkpatrick P, Ritchie A, Goddard M, Bennett M. Vascular smooth muscle cells undergo telomere-based senescence in human atherosclerosis: effects of telomerase and oxidative stress. Circ Res, 2006, 99: 156-164

6 Minamino T, Miyauchi H, Yoshida T, Komuro I. Endothelial cell senescence in human atherosclerosis: role of telomere in endothelial dysfunction. Circulation, 2002, 105: 1541-1544

7 Okuda K, Bardeguez A, Gardner JP, Rodriguez P, Ganesh V, Kimura M, Skurnick J, Awad G, Aviv A. Telomere length in the newborn. Pediatr Res, 2002, 52: 377-381

8 Hewitt G, Jurk D, Marques FD, Correia-Melo C, Hardy T, Gackowska A, Anderson R, Taschuk M, Mann J, Passos JF. Telomeres are favored targets of a persistent DNA damage response in ageing and stress-induced senescence. Nat Commun, 2012, 3: 708

9 Ogami M, Ikura Y, Ohsawa M, Matsuo T, Kayo S, Yoshimi N, Hai E, Shirai N, Ehara S, Komatsu R, Naruko T, Ueda M. Telomere shortening in human coronary artery diseases. Arterioscler Thromb Vasc Biol, 2004, 24: 546-550

10 Chang E, Harley CB. Telomere length and replicative aging in human vascular tissues. Proc Natl Acad Sci USA, 1995, 92: 11190-11194

11 Brouilette SW, Moore JS, McMahon AD, Thompson JR, Ford I, Shepherd J, Packard CJ, Samani NJ; West of Scotland Coronary Prevention Study Group. Telomere length, risk of coronary heart disease, and statin treatment in the West of Scotland Primary Preven- tion Study: a nested case-control study. Lancet, 2007, 369: 107-114

12 O'onnell CJ, Demissie S, Kimura M, Levy D, Gardner JP, White C, D'gostino RB, Wolf PA, Polak J, Cupples LA, Aviv A. Leukocyte telomere length and carotid artery intimal medial thickness: the Framingham Heart Study. Arterioscler Thromb Vasc Biol, 2008, 28: 1165-1171

13 Willeit P, Willeit J, Brandstätter A, Ehrlenbach S, Mayr A, Gasperi A, Weger S, Oberhollenzer F, Reindl M, Kronenberg F, Kiechl S. Cellular aging reflected by leukocyte telomere length predicts advanced atherosclerosis and cardiovascular disease risk. Arterioscler Thromb Vasc Biol, 2010, 30: 1649-1656

14 Zhang W, Chen Y, Wang Y, Liu P, Zhang M, Zhang C, Hu FB, Hui R. Short telomere length in blood leukocytes contributes to the presence of atherothrombotic stroke and hemorrhagic stroke and risk of post-stroke death. Clin Sci, 2013, 125: 27-36

15 Kimura M, Hjelmborg JB, Gardner JP, Bathum L, Brimacombe M, Lu X, Christiansen L, Vaupel JW, Aviv A, Christensen K. Short leukocyte telomeres forecast mortality: a study in elderly Danish twins. Am J Epidemiol, 2008, 167: 799-806

16 Aviv A, Chen W, Gardner JP, Kimura M, Brimacombe M, Cao X, Srinivasan SR, Berenson GS. Leukocyte telomere dynamics: longitudinal findings among young adults in the Bogalusa Heart Study. Am J Epidemiol, 2009, 169: 323-329

17 Xiao Q, Kiechl S, Patel S, Oberhollenzer F, Weger S, Mayr A, Metzler B, Reindl M, Hu Y, Willeit J, Xu Q. Endothelial progenitor cells, cardiovascular risk factors, cytokine levels and atherosclerosis-results from a large population-based study. PLoS ONE, 2007, 2: e975

18 Andrew T, Aviv A, Falchi M, Surdulescu GL, Gardner JP, Lu X, Kimura M, Kato BS, Valdes AM, Spector TD. Mapping genetic loci that determine leukocyte telomere length in a large sample of unselected female sibling pairs. Am J Hum Genet, 2006, 78: 480-486

19 Armanios M, Blackburn EH. The telomere syndromes. Nat Rev Genet, 2012, 13: 693-704

20 Levy D, Neuhausen SL, Hunt SC, Kimura M, Hwang SJ, Chen W, Bis JC, Fitzpatrick AL, Smith E, Johnson AD, Gardner JP, Srinivasan SR, Schork N, Rotter JI, Herbig U, Psaty BM, Sastrasinh M, Murray SS, Vasan RS, Province MA, Glazer NL, Lu X, Cao X, Kronmal R, Mangino M, Soranzo N, Spector TD, Berenson GS, Aviv A. Genome-wide association identifies $\mathrm{OBFC} 1$ as a locus involved in human leukocyte telomere biology. Proc Natl Acad Sci USA, 2010, 107: 9293-9298

21 Codd V, Mangino M, van der Harst P, Braund PS, Kaiser M, Beveridge AJ, Rafelt S, Moore J, Nelson C, Soranzo N, Zhai G, Valdes AM, Blackburn H, Mateo Leach I, de Boer RA, Kimura M, Aviv A; Wellcome Trust Case Control Consortium, Goodall AH, Ouwehand W, van Veldhuisen DJ, van Gilst WH, Navis G, Burton PR, Tobin MD, Hall AS, Thompson JR, Spector T, Samani NJ. Common variants near TERC are associated with mean telomere length. Nat Genet, 2010, 42: 197-199

22 Li S, Makovets S, Matsuguchi T, Blethrow JD, Shokat KM, Blackburn EH. Cdk1-dependent phosphorylation of Cdc13 coordinates telomere elongation during cell-cycle progression. Cell, 2009, 136: 50-61

23 Eash KJ, Means JM, White DW, Link DC. CXCR4 is a key regulator of neutrophil release from the bone marrow under basal and stress granulopoiesis conditions. Blood, 2009, 113: 4711-4719

24 Sainz J, Sata M. Open sesame! CXCR4 blockade recruits neutrophils into the plaque. Circ Res, 2008, 102: 154-156

25 Myocardial Infarction Genetics Consortium. Genome-wide association of early-onset myocardial infarction with single nucleotide polymorphisms and copy number variants. Nat Genet, 2009, 41: 334-341

26 Boccardi V, Paolisso G. Telomerase activation: a potential key modulator for human healthspan and longevity. Ageing Res Rev, 2014, $15 \mathrm{C}: 1-5$ 
27 Concetti F, Carpi FM, Nabissi M, Picciolini M, Santoni G, Napolioni V. The functional polymorphism rs73598374:G>A (p.Asp8Asn) of the ADA gene is associated with telomerase activity and leukocyte telomere length. Eur J Hum Genet, 2014, doi: 10.1038/ejhg.2014.102

28 Yu TY, Kao YW, Lin JJ. Telomeric transcripts stimulate telomere recombination to suppress senescence in cells lacking telomerase. Proc Natl Acad Sci USA, 2014, 111: 3377-3382
29 Frenck RW Jr, Blackburn EH, Shannon KM. The rate of telomere sequence loss in human leukocytes varies with age. Proc Natl Acad Sci USA, 1998, 95: 5607-5610

30 Matthews C, Gorenne I, Scott S, Figg N, Kirkpatrick P, Ritchie A, Goddard M, Bennett M. Vascular smooth muscle cells undergo telomere-based senescence in human atherosclerosis: effects of telomerase and oxidative stress. Circ Res, 2006, 99: 156-164

Open Access This article is distributed under the terms of the Creative Commons Attribution License which permits any use, distribution, and reproduction in any medium, provided the original author(s) and source are credited. 